\title{
Design of portable emergency lamp utilizing thin film solar cell and inflatable case
}

\author{
Andrew Bastian ${ }^{1}$, Kresna Devara ${ }^{1}$, Savira Ramadhanty ${ }^{1}$, and Tomy Abuzairi ${ }^{1, *}$ \\ ${ }^{1}$ Department of Electrical Engineering, Faculty of Engineering, Universitas Indonesia, Kampus Baru UI Depok 16424, Indonesia
}

\begin{abstract}
Lighting is an essential thing in performing daily activities and without sufficient lighting we will be difficult to see clearly. The problem is when there is no electricity, for example when we go to a cave or a forest or when there is a natural disaster that shuts down all the electricity. A portable lamp that can be charged by exposing the lamp to sun light can be one of the solution to overcome this problem. Energy Harvesting is a concept where an energy is captured, stored, and used with several technologies including solar technology. Energy Harvesting technology is used in many applications such as calculator, electrical cars or day-to-day lighting This paper will discuss about an application of solar panel in portable LED lamp, that can be used in the night and can be charged during daytime with solar panel. The solar panel, LED, and the battery is integrated in one circuit so there will be more space convenient for user. Solar emergency lamp can be used to illuminate the pathway in mountain or caves and the lamp can float in the water because it case is inflatable. Rechargeable Li-ion Battery $3.7 \mathrm{~V} 500 \mathrm{mAh}$ and 10 LED SMD is used in this lamp. The proposed design is using a transparent PVC for the case of the lamp. The lamp can be turned on approximately for 4 hours from fully charged battery with 10 LED lamp. The illumination of solar portable emergency lamp was measured using lux meter. The illumination average of the solar portable emergency lamp is 17.58 lux with a deviation of 7.3 lux with medium bright mode. The illumination average of the solar portable emergency lamp is 32.85 lux with a deviation of 12.4 lux for the full bright mode. The Illumination is measured in $50 \mathrm{~cm}$ below the solar portable emergency lamp with 9 measured point consisting of 3 rows and 3 columns.
\end{abstract}

\section{Introduction}

Energy harvesting is a concept where an energy is captured, stored, and used with several technologies including solar technology [1]. Solar Technology can convert photon energy to electricity and called a Photovoltaic concept. Photovoltaic is a concept of converting photon energy from sunlight to electricity using semiconductor material that can be used for charging a battery [2]. Energy Harvesting can be an alternative for small devices such as a lamp or battery powered device because the battery doesn't have to be replaced if its empty.

Energy harvesting technology is used in many applications such as calculator, electrical cars or day-today lighting [1]. There is so much solar product especially in solar lamp that used in the street, or used to powered lamp for indoor lighting. There are a few products that using solar technology integrated with LED lamp for a portable solar lamp that can be used for daily activities.

Lighting is an essential thing in performing daily activities and without sufficient lighting we will be difficult to see clearly. Sufficient lighting can be helpful in nature with dark environment like in the cave or in the forest. The problem is when there is no electricity, for example when we go to a cave or a forest or when there is a natural disaster that shuts down all the electricity. A portable lamp can be one of the solution to overcome this problem such as kerosene lamp but kerosene lamp is emitting black carbon (BC) that are not good for health [3]. So, a renewable energy lamp such as solar powered lamp is an alternative solution to overcome this problem.

In this paper, we present a solar technology integrated with LED lamp and battery that can be used for portable lamp. Usually, an energy harvesting system consist of solar panel, battery, and LED [4]. The portable lamp can be charged during daytime and be used to giving lighting assistant during daytime or night time. Since the lamp is integrated with solar panel and battery, it will be convenient and lightweight to carried around. Especially when hiking, we don't want to bring something heavy and spacious. The lamp is made inflatable to be able to float in water and the lamp can be deflated to fit into pockets. The difference between ordinary flashlight and inflatable solar lamp are described in Table 1.

\footnotetext{
Corresponding author: tomy@ee.ui.ac.id
} 
Table 1. Differences between ordinary flashlight and proposed solar lamp.

\begin{tabular}{|c|c|c|}
\hline Differences & Flashlight & Inflatable Solar Lamp \\
\hline Lighting & $\begin{array}{c}\text { Focus on } 1 \\
\text { spot }\end{array}$ & Area \\
\hline Float & No & Yes \\
\hline Weight & Heavier & Lighter \\
\hline Rechargeable & No & Yes \\
\hline Shape & Cylindrical & Cube (if inflated $8 \times 8 \times 1 \mathrm{~cm})$ \\
\hline Cost & $\sim \$ 4.00$ & Less than $\$ 4.00$ \\
\hline
\end{tabular}

Solar powered lamp system provides an easy to deploy lamp and minimal maintenance. The solar cell can charge the battery so that there is no need for operational cost. The system is consists of a solar panel, a battery, a LED, and a chip controller. The chip controller is giving the lamp a 3 mode of operation. The first operation is a full bright mode, the second is a medium bright mode, and the third is the flicker mode.

\section{Design of Solar Portable Emergency Lamp}

Solar portable emergency lamp integrating all of the components into one circuit. The components consist of solar panel, LED, battery, and controller. The top of the device is solar panel, while the bottom one are the LED, battery, and controller.

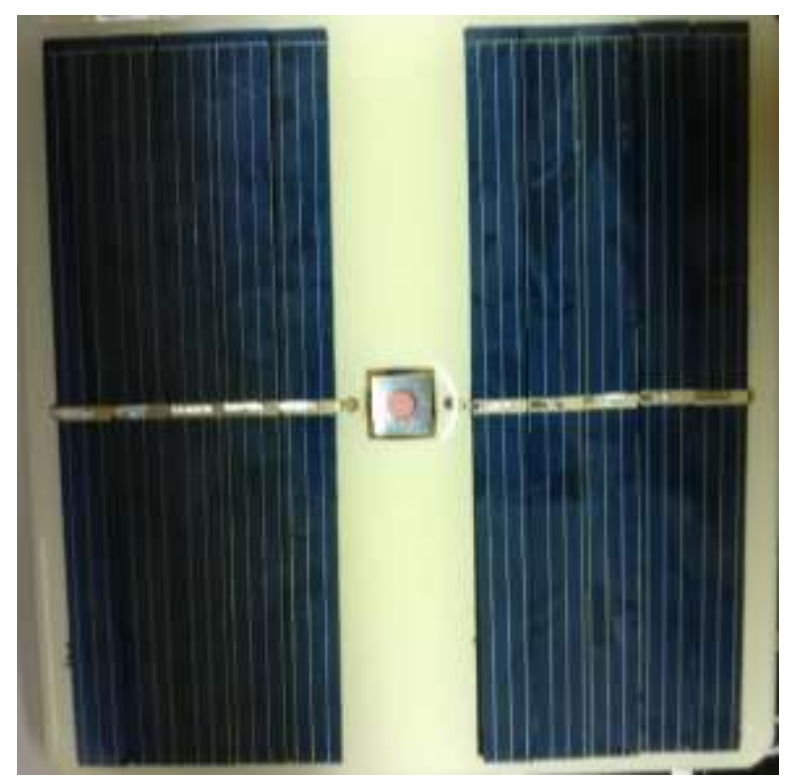

Fig. 1. Top view of the solar emergency lamp circuit

Figure 1 shows top view of the solar emergency lamp. Solar panel is utilized to absorb light and convert it to electricity [5]. The integrated circuit is cased with transparent PVC and made to inflate so the lamp can float in water. The circuit dimension is $8 \times 8 \times 0.5 \mathrm{~cm}$. On the top of the circuit, the solar panel will power the lamp and charge the battery. There is a switch on the center of the solar panel to switch from 3 mode operation

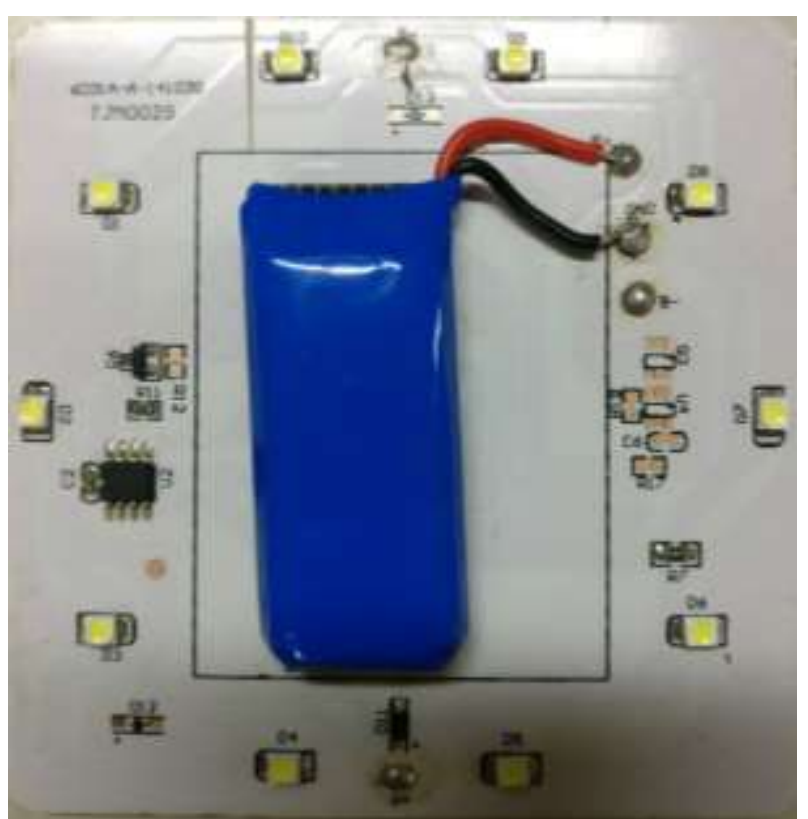

Fig. 2. Bottom view of the solar emergency lamp circuit

Figure 2 shows bottom view of the solar emergency lamp. The bottom of the circuit consist of LED, battery and controller. Rechargeable Lithium-ion Battery 3.7 V $500 \mathrm{mAh}$ and 10 LED SMD is used in this lamp. Lithium-ion is utilized due to their high energy density, high power density, long service life and environmental friendliness [6]. The controller is triggered by the switch on the top of the circuit and can change to 3 mode of operation.

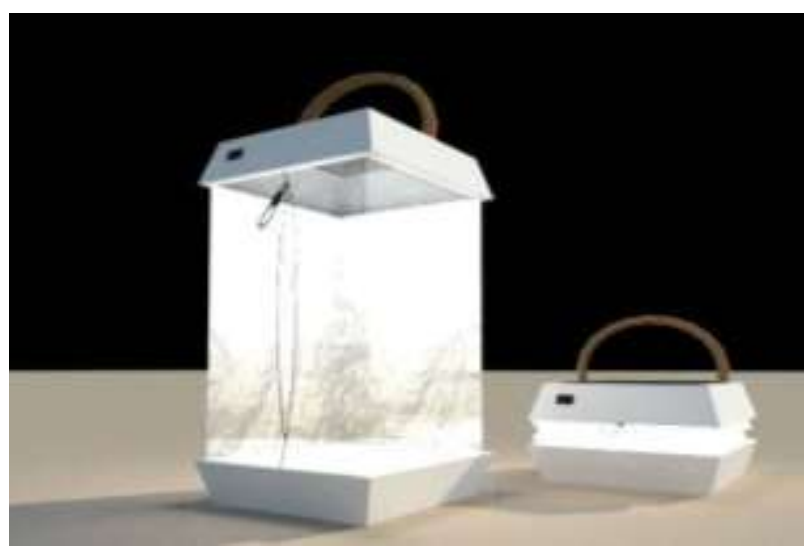

Fig. 3. Inflatable case of solar emergency lamp

The case is made inflatable to improve portability because user can squeeze the case into a flat case for space convenient as shown in Figure 3.. The inflatable case can float in water so user will have lighting when they go to caves and the lamp can be hooked on bags so it can give lighting to the path. The case is made of transparent PVC so the light from the LED can illuminate the surrounding. The PVC will provide a glare-free lighting to the surrounding that are suitable for camping, reading at night and walking at night. 


\section{Working principle of Solar Portable Emergency Lamp}

\subsection{Circuit}

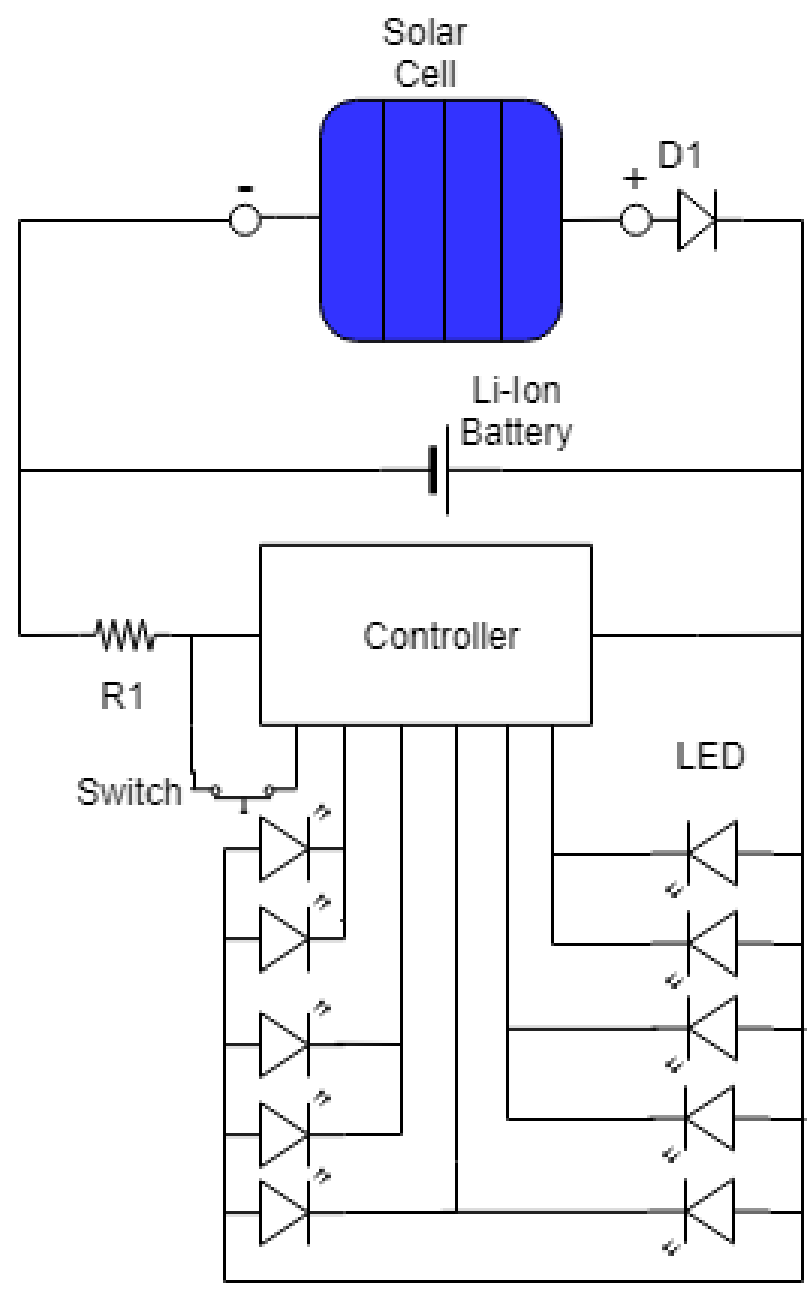

Fig. 4. Circuit of solar emergency lamp

The main component for this portable solar lamp consist of: Solar cell, Li-Ion Battery, Controller and LED, as shown in Figure 4. Solar cell is used to absorb solar energy radiation and convert it to electricity. Polysilicon solar cell is used in this solar lamp with maximum power is $0.55 \mathrm{~W}$ and efficiency around $10 \%$. Solar cell will charge the Li-Ion Battery when exposed to sunlight and the electricity will flow to the diode to the battery. The diode is used to block electricity from the battery to the solar cell so the solar cell wont broke. The battery is charged when the solar panel voltage is higher than the remaining voltage of the battery.

The LED is connected to the controller on 5 ports with 2 parallel LED in each port. This configuration allows the controller to split the current evenly to each port so the LED will bright evenly. The switch will trigger the controller to do 3 modes of operation, and the $\mathrm{R} 1$ used for pull down resistor. The solar panel is integrated with the LED and the battery to reduce the space for portability. This portable lamp is using Li-Ion $3.7 \mathrm{~V} 500 \mathrm{mAh}$ battery that can powered the lamp for approximately 4 hours.

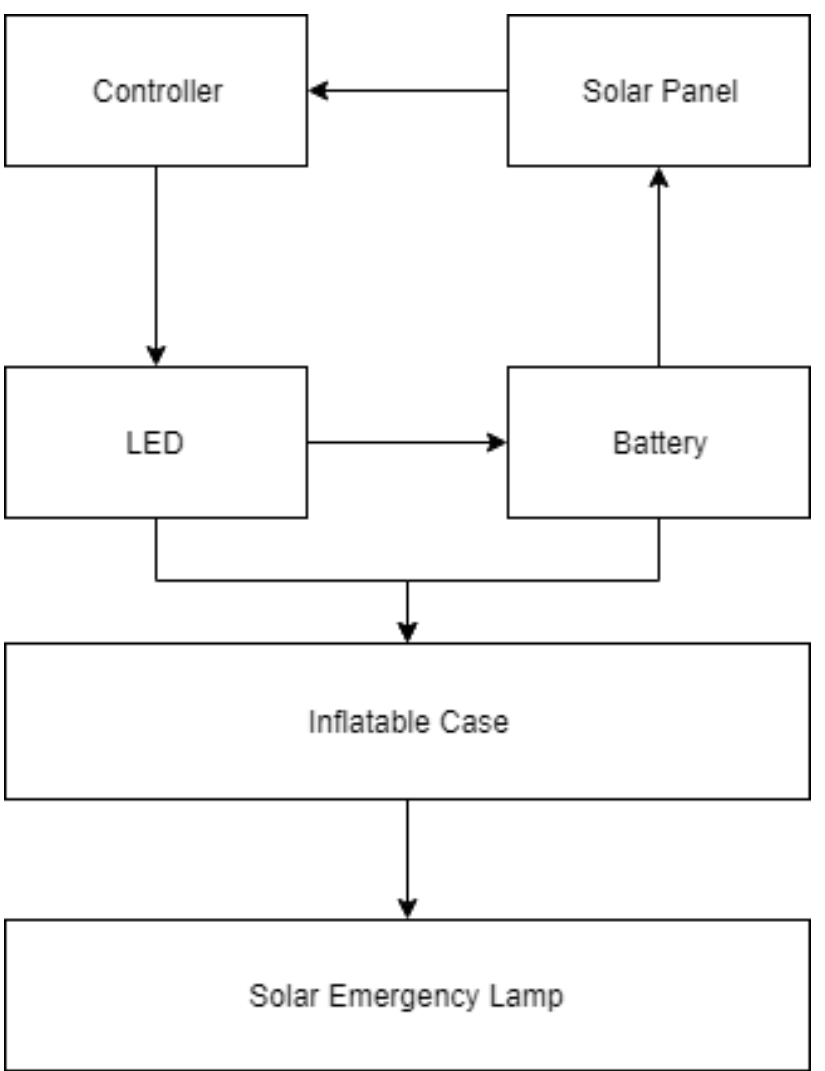

Fig. 5. Structure Frame of the Solar Emergency Lamp

The component for Solar Emergency Lamp consists of solar panel, LED, battery, and controller. The components are integrated on 1 circuit as shown in Fig. 2 and cased with transparrent PVC to make the case inflatable. The top of the circuit are the solar panel and the bottom is the controller, battery, and LED, as shown in Fig. 3 and Fig. 4.

\section{Results and Discussion}

Table 2. Mode of Operation Result.

\begin{tabular}{|c|c|c|c|}
\hline $\begin{array}{l}\text { Mode of } \\
\text { Operation }\end{array}$ & $\begin{array}{l}\text { Voltage } \\
\text { (V) }\end{array}$ & $\begin{array}{l}\text { Current } \\
(\mathrm{mA})\end{array}$ & $\begin{array}{l}\text { Power } \\
\text { (Watt) }\end{array}$ \\
\hline Full Bright & 3.8 & 124.7 & 0.47 \\
\hline Medium Bright & 3.8 & 62.9 & 0.24 \\
\hline Flicker & 3.8 & 124.7 & 0.47 \\
\hline
\end{tabular}

The power needed to operate the lamp is calculated using

$$
\mathrm{P}=\mathrm{V} \times \mathrm{I}
$$

If the battery is full, it has a $3.7 \mathrm{~V}$ and $500 \mathrm{mAh}$ so the energy of the battery is 1.85 Watt-Hour that calculated using:

$$
\mathrm{E}=\mathrm{V} \times \mathrm{I} \times \mathrm{t}
$$

So, the lamp can be turned on for 3.94 Hour ( $\cong 4$ Hour) by using:

$$
\mathrm{T}=\mathrm{E} \div \mathrm{P}
$$


The intensity of the solar portable emergency lamp is measured in a dark room with a scheme shown in Figure 6 . The measurement point is consisting of 3 rows and 3 columns and total of 9 point. The distance from each row and column is $30 \mathrm{~cm}$ and the solar portable emergency lamp is measured from $50 \mathrm{~cm}$ height with a lux meter.

The illumination for the medium bright mode is shown in Fig.7. The highest illumination is achieved with 36.14 lux because the point is below the lamp in the second row of the second column. The illumination dropped to 11.62 in the first column of the third row. Average illumination of medium bright mode is 17.58 lux with a deviation of 7.3 lux. Moreover, the illumination for the high bright mode is shown in Fig.8. The highest illumination is achieved with 64.40 lux because the point is below the lamp in the second row of the second column. The illumination dropped to 21.09 in the third column of the third row. Average illumination of full bright mode is 32.85 lux with a deviation of 12.4 lux. From the measurement, the illumination different between medium and full bright mode is $\sim 30$ lux.

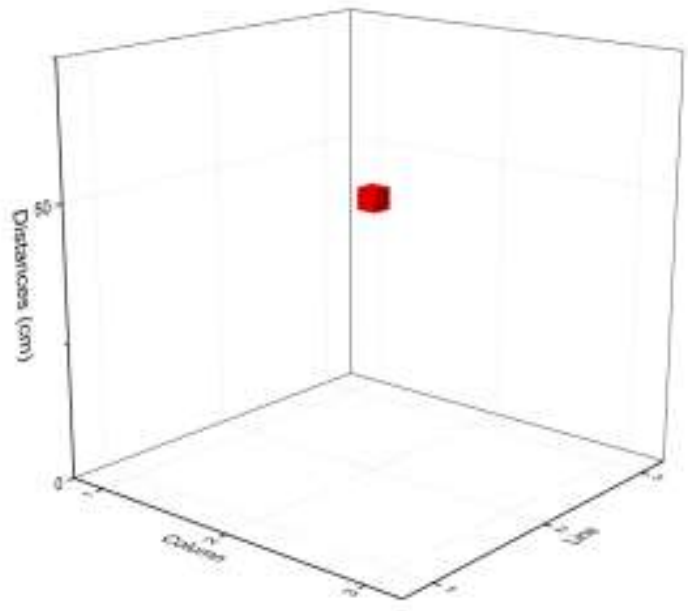

Fig. 6. Measurement position of solar portable emergency lamp.

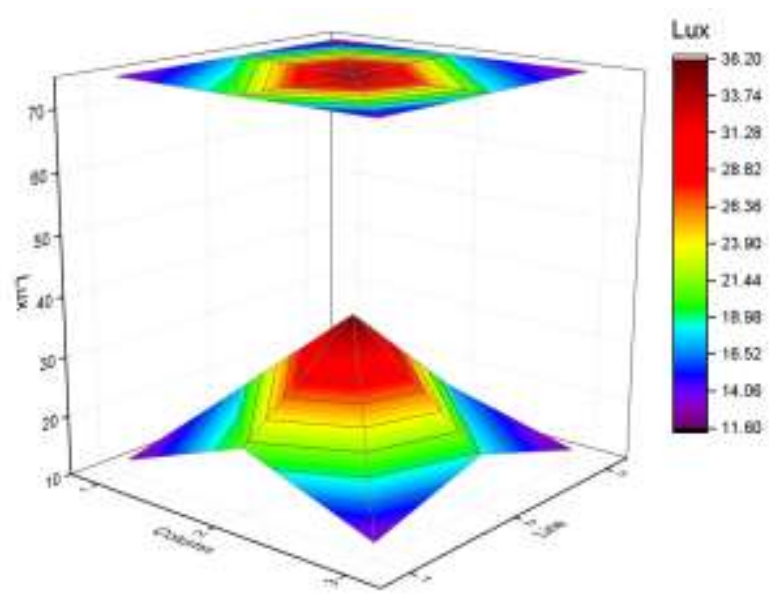

Fig. 7. Illumination measurement of medium bright mode.

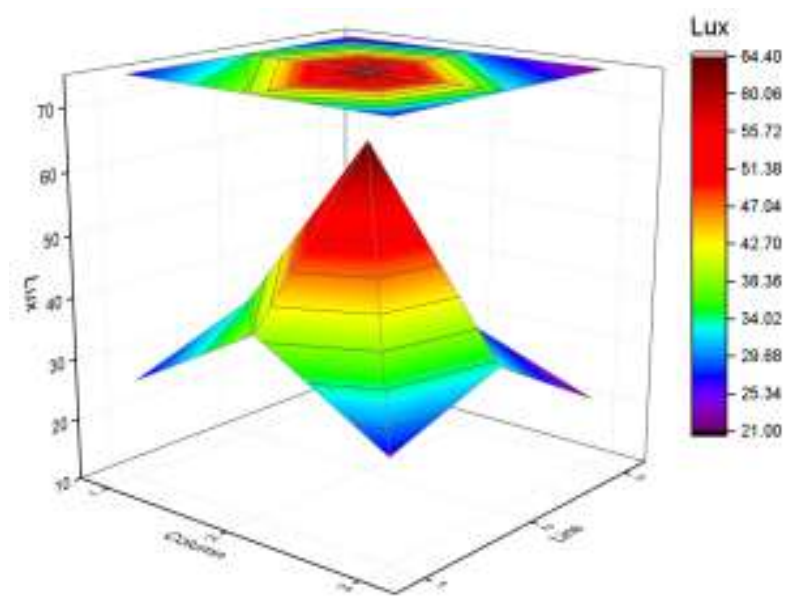

Fig. 8. Illumination measurement of full bright mode.

\section{Conclusion}

Solar emergency lamp has been successfully designed. The solar emergency lamp has an inflatable case for portability purposes because it can be squeeze up to save space. The lamp can be turned on with full bright mode for approximately 4 hours with full charged battery with 10 LED lamp.

The illumination of solar portable emergency lamp was measured using lux meter. The illumination average of the solar portable emergency lamp is 17.58 lux with a deviation of 7.3 lux with medium bright mode. The illumination average of the solar portable emergency lamp is 32.85 lux with a deviation of 12.4 lux for the Full bright mode. The Illumination is measured in $50 \mathrm{~cm}$ below the solar portable emergency lamp with 9 measured point consisting of 3 rows and 3 columns.

This work has been supported by International publications indexed for the Final Project of Students (No. 2549/UN2.R3.1/HKP.05.00/2018) Research Grant 2018 from Universitas Indonesia.

\section{References}

1. A. Khaligh and O.C. Onar, Energy Harvesting: solar, wind, and ocean energy conversion systems (CRC Press, pp. xiii-1, 2017)

2. A. L. Fahrenbruch and R. H. Bube, Fundamentals in Solar Cells (Academic Press, 1983)

3. N.L. Lam, Y. Chen, C. Weyant, C. Venkataraman, P. Sadavarte, M.A. Johnson, K.R. Smith, B.T. Brem, J. Arineitwe, J.E. Ellis, and T.C. Bond. Household light makes global heat: high black carbon emissions from kerosene wick lamps. Environmental science \& technology, 46(24), pp.13531-13538, (2012)

4. A. Nasiri, S. A. Zabalawi, and G. Mandic. Indoor Power Harvesting Using Photovoltaic Cells for Low-Power Applications. (IEEE Transactions on Industrial Electronics, Vol 56, no. 11, pp. 45024509, 2009) 
5. M. A. Green, Solar cells: operating principles, technology, and system applications (Englewood Cliffs, NJ, Prentice-Hall, Inc., 1982)

6. Lu, L., Han, X., Li, J., Hua, J., \& Ouyang, M.. A review on the key issues for lithium-ion battery management in electric vehicles. Journal of power sources, 226, 272-288 (2013) 